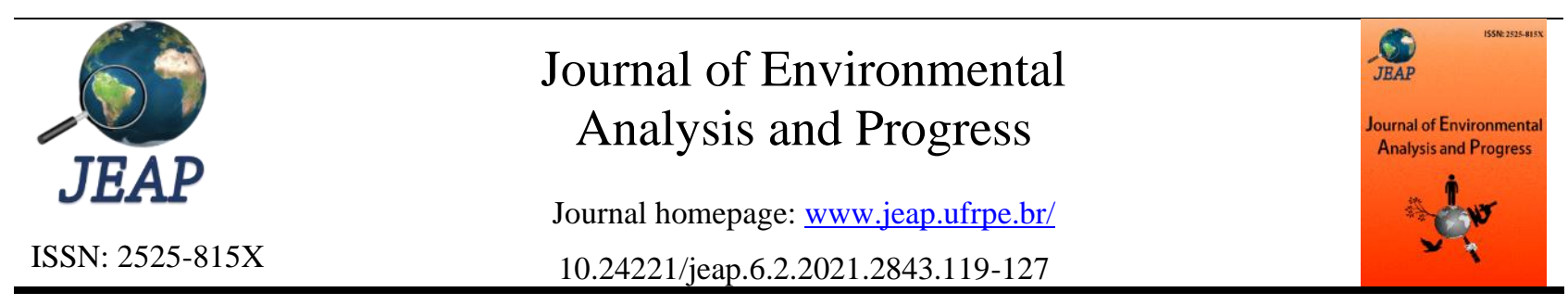

\title{
Aplicação de sensores de baixo custo na estimativa da evapotranspiração potencial
}

\section{Application of low-cost sensors to estimate potential evapotranspiraton}

\author{
Ailton Alves de Carvalho ${ }^{a}$, Victor Wanderley Costa de Medeiros ${ }^{\mathrm{a}}$, Glauco Estácio Gonçalves ${ }^{\mathrm{a}}$ \\ ${ }^{a}$ Universidade Federal Rural de Pernambuco-UFRPE, Departamento de Engenharia Agrícola. Rua Dom Manoel de \\ Medeiros, s/n, Dois Irmãos, Recife, PE, Brasil. CEP: 52.171-900. E-mail: ailtonalvesst@hotmail.com (*Autor \\ correspondente), victor.wanderley@ufrpe.br, glaestgon@gmail.com.
}

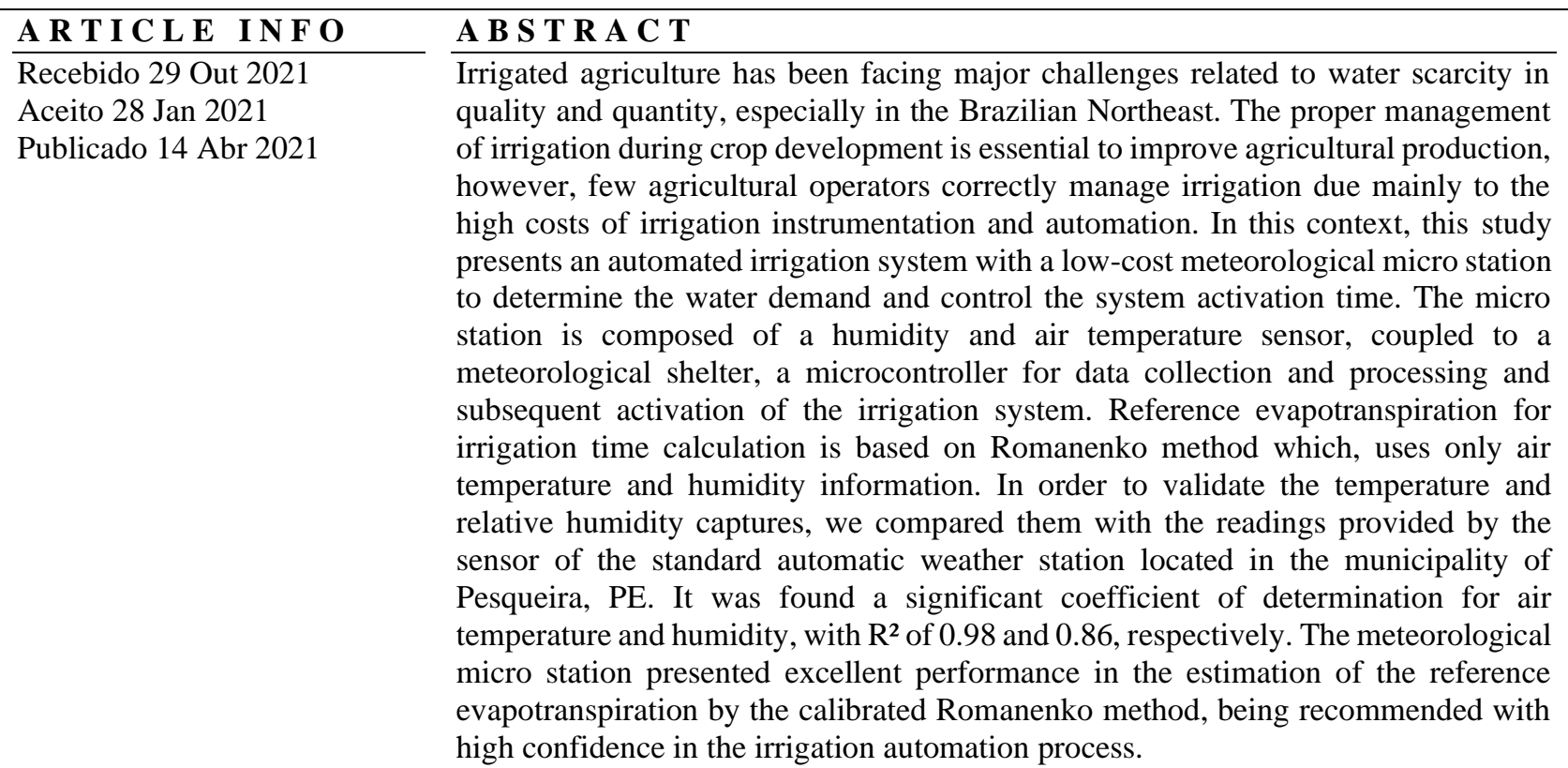

Keywords: Humidity sensor, air temperature, water efficiency.

\section{R E S U M O}

A agricultura irrigada vem enfrentando grandes desafios relacionados à escassez de água em qualidade e quantidade, principalmente no Nordeste brasileiro. O manejo adequado da irrigação durante o desenvolvimento da cultura é essencial para melhorar a produção agrícola, porém, poucos produtores rurais realizam o manejo de irrigação de forma adequada devido, principalmente, aos custos elevados da instrumentação e automação da irrigação. Neste contexto, este estudo apresenta um sistema de irrigação automatizado que utiliza uma microestação meteorológica de baixo custo para determinação da demanda de água e controle do tempo de acionamento do sistema. A microestação é composta por um sensor de umidade e temperatura do ar, acoplado a um abrigo meteorológico, um microcontrolador para coleta e processamento dos dados e posterior acionamento do sistema de irrigação. A evapotranspiração de referência para o cálculo do tempo de irrigação é baseada no método de Romanenko que utiliza apenas informações de temperatura e umidade do ar. Para validação das capturas de temperatura e umidade relativa do ar, realizou-se comparação das leituras fornecidas pelo sensor da estação meteorológica automática padrão localizada no Município de Pesqueira, PE. Constatou-se um ótimo coeficiente de determinação para a temperatura e umidade do ar, com $\mathrm{R}^{2}$ de 0,98 e 0,86 , respectivamente. A microestação meteorológica apresentou ótimo desempenho na estimativa da evapotranspiração de referência pelo método de Romanenko calibrado, 
sendo recomendada com alto grau de confiança no processo de automação de irrigação.

Palavras-Chave: Sensor de umidade, temperatura do ar, eficiência hídrica.

\section{Introdução}

Em regiões áridas e semiáridas conhecer a quantidade de água necessária pela cultura é fundamental num programa de manejo de irrigação, visto que a água é fator escasso durante maior parte do ano. Um sistema de irrigação adequado deve melhorar a eficiência de uso da água, aumentando a produtividade das culturas e, simultaneamente, proporcionar maior retorno dos investimentos.

Os sistemas de irrigação controlados manualmente geralmente não garantem o nível adequado de água na área irrigada, podendo ocorrer tanto o desperdício pela irrigação em demasia (encharcamento), quanto a diminuição da produtividade quando não é fornecido a uma determinada cultura a quantidade de água necessária para suprir sua necessidade (Agrawal et al., 2018).

Os sistemas de irrigação automáticos geralmente são projetados para garantir o nível adequado de água de acordo com o crescimento das plantas durante toda fase de cultivo, mesmo sem a presença de operadores (Uddin et al., 2012).

Conhecer a quantidade de água requerida pelas culturas é de grande importância na agricultura irrigada para a realização de um adequado programa de manejo de irrigação (Lopes et al., 2004). Com um manejo adequado, um sistema de irrigação deve proporcionar maior eficiência, aumentando a produtividade das culturas, diminuindo os custos de produção e, consequentemente, proporcionando maior retorno dos investimentos (Duarte, 2006).

O sucesso do uso de água para irrigação depende, entre outros requisitos, do conhecimento preciso da demanda hídrica da cultura. Assim, aplicando-se o manejo via clima, torna-se necessário o uso de coeficientes adequados, especificamente o coeficiente da cultura $(\mathrm{Kc})$, determinado com base na evapotranspiração da cultura (ETc) e evapotranspiração de referência (ETo), cujas estimativas permitem a avaliação da quantidade de água a ser aplicada em cada uma delas (Melo et al, 2013).

No entanto, o monitoramento climático associado à automação da irrigação apresenta, em geral, um custo inviável para os agricultores familiares. De acordo com Uddin et al. (2012), devido aos altos custos de sistemas de irrigação automatizados, os agricultores em geral (pequenos e médios) não podem comprá-los para uso, ficando restrito às fazendas apenas para experimentos ou demonstrações financiadas pelo governo, ou por organizações privadas.

Nos últimos anos, de acordo com relatórios oficiais, para produzir os melhores produtos em qualidade, quantidade e condições econômicas viáveis, é recomendado que o setor agrícola se beneficie com novas ferramentas e técnicas provenientes do campo das Tecnologias de Informação e Comunicação (TIC). Dessa forma, durante a última década, a implantação de dispositivos sensores aumentou consideravelmente no campo da agricultura. Esse fato levou a um novo conceito chamado agricultura inteligente $\mathrm{e}$ contempla atividades como monitoramento de campo, que oferecem suporte para a tomada de decisões ou a realização de ações, como irrigação ou fertilização (Trilles et al., 2019).

Sistemas de irrigação por gotejamento automático que regam as plantas com base na umidade do solo, valor de $\mathrm{pH}$ do solo, temperatura e luz são utilizados por outros pesquisadores (Agrawal \& Singhal, 2015). Porém, falta conhecimento do comportamento dos sensores em diversas condições climáticas. Nas condições climáticas de Mato Grosso, Silva Filho et al. (2019) trabalharam com sensores de baixo custo que foram calibrados para aquela região. Os autores encontraram excelente comportamento diário, permitindo o uso dos equipamentos em campo.

O desenvolvimento e testes em campo de soluções como esta, possibilita validar e consolidar a tecnologia de instrumentação desenvolvida, com destaque para as de custos reduzidos e minimiza a dependência de importação de equipamentos (Silva Filho et al., 2019).

Neste contexto, este estudo apresenta um sistema automático de irrigação de baixo custo que, baseado na medida de temperatura e umidade do ar, determina a lâmina de irrigação a ser aplicada à cultura. Este mesmo sistema, comanda, automaticamente, a(s) motobomba(s), iniciando e finalizando o processo de irrigação.

\section{Material e Métodos}

Descrição do sistema de irrigação

O sistema automático de irrigação é composto por uma microestação meteorológica, fonte de energia para alimentação da microestação e alimentação de motobomba com acionamento via relé e uma fonte hídrica, composta de uma caixa d'água e o sistema de distribuição de água (gotejo). A Figura 1 ilustra como estes componentes são interconectados. 


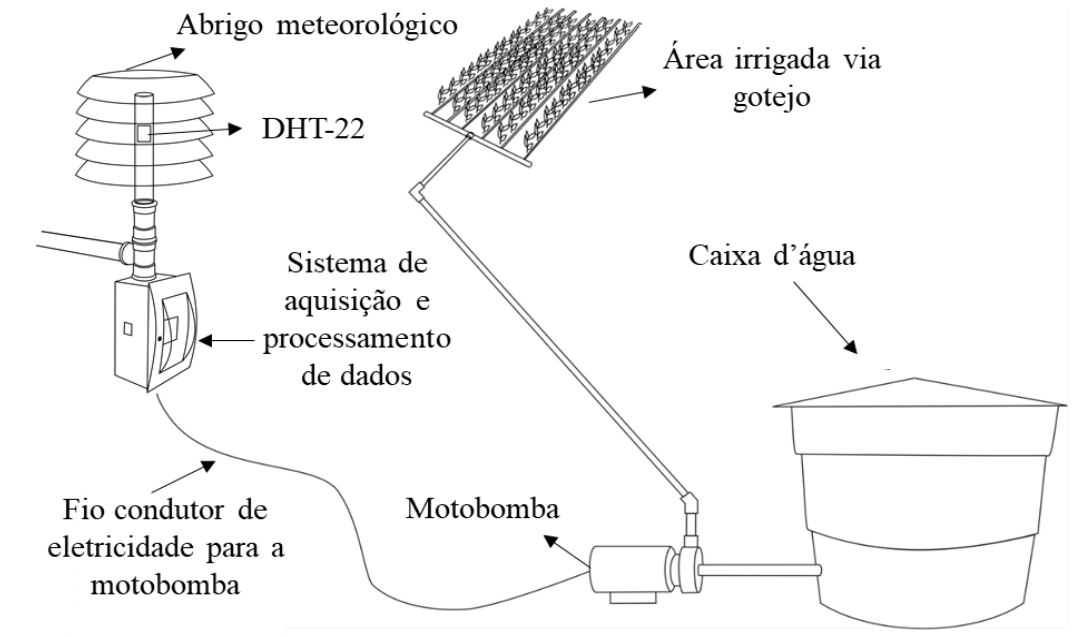

Figura 1. Componentes do sistema de irrigação. Fonte: Carvalho et al. (2020).

A microestação meteorológica (Figura 2) é composta por um abrigo meteorológico que comporta o sensor de temperatura e umidade relativa do ar. Este abrigo é conectado à caixa de coleta e processamento de dados, que abriga um microcontrolador (NodeMCU - ESP8266), um relé para acionamento da motobomba, circuito de relógio, circuito para armazenamento de dados e dois botões para interação com o produtor rural.

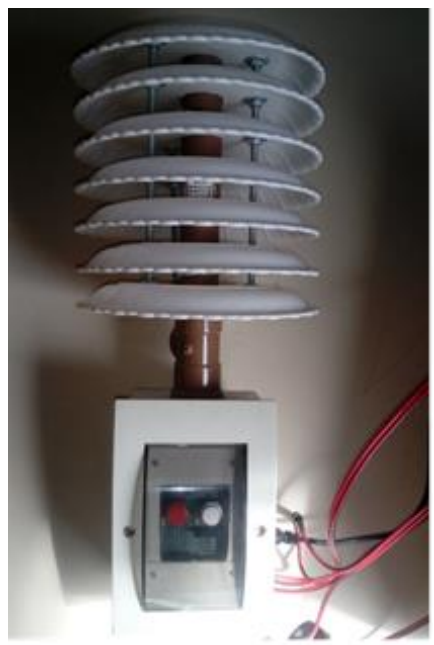

Figura 2. Imagem da microestação montada para teste. Fonte: Carvalho et al. (2020).

O botão branco serve para inicializar o sistema de irrigação (Figura 3). Após iniciado, o mesmo botão permite o acionamento manual da motobomba, que pode ser usado em caso de falha do sensor de coleta dos dados, ou outra situação que o produtor julgar necessária. Quando há uma falha do sensor de coleta dos dados, um aviso luminoso indica ao produtor a situação para que este possa fazer o acionamento manual da motobomba e garantir que a cultura será irrigada. $\mathrm{O}$ desligamento da motobomba é feito pelo botão vermelho.

Em cenário de operação normal, o botão vermelho evita a irrigação automática por um dia. Este botão deve ser usado quando o produtor observa a ocorrência de precipitação igual ou superior a $8 \mathrm{~mm}$, como especificado por Pereira Filho et al. (2010) para as fases de elevada demanda, a exemplo, da cultura do milho $(7,5 \mathrm{~mm}$ diários), ou em qualquer outra necessidade. Para manter seu custo mais baixo, a microestação meteorológica não possui um pluviômetro acoplado. Portanto, o produtor deve possuir pluviômetro para determinação da precipitação, que pode ser confeccionado com o uso de uma garrafa PET, por exemplo.

O código-fonte para funcionamento do sistema de irrigação está disponível em https://github.com/jualabs/iot/tree/master/JuaSISP rotoA.

A estação simplificada é composta por um sensor DHT22, que fornece a temperatura e umidade do ar com precisão de $0,5{ }^{\circ} \mathrm{C}$ e $2 \%$, respectivamente, sendo este sensor protegido por abrigo meteorológico. Estes sensores são lidos há cada 15 minutos, com valores médios registrados a cada hora para posterior cálculo da evapotranspiração de referência (ETo) diária. Os dados da evapotranspiração do dia anterior são utilizados para o cálculo do tempo de irrigação a cada dia. 


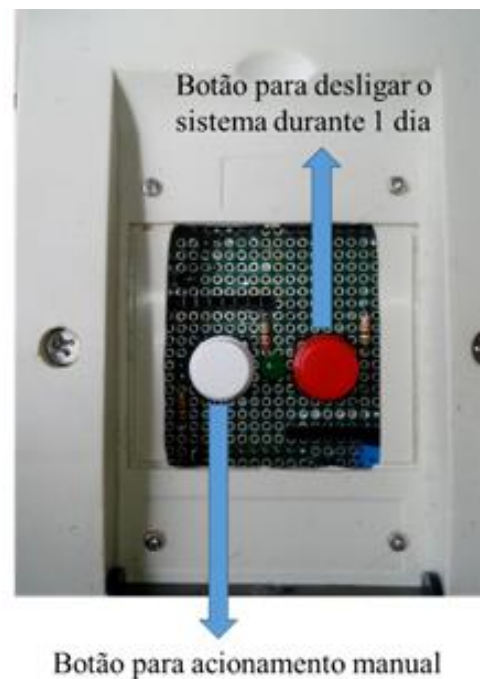

Figura 3. Botões de acionamento da microestação montada para teste. Fonte: Carvalho et al. (2020).

Na caixa de proteção do microcontrolador NodeMCU, também está presente um relógio de tempo real - RTC (Real Time Clock), para manutenção precisa da hora de irrigação e dos tempos de coleta dos dados, e um módulo de cartão $\mathrm{SD}$, para armazenamento das informações coletadas. Além disso, existe um relé que aciona a motobomba de acordo com o tempo de irrigação determinado pelo sistema.

A estação é alimentada por uma fonte de energia externa, que também acionará a motobomba de forma automática pela estação, de acordo com o cálculo do tempo de irrigação.

Informações iniciais a respeito do sistema de irrigação, do cultivo e do solo são necessários antes da implantação do sistema.

A estação simplificada toma como base informações de evapotranspiração da cultura (ETc), do coeficiente de cultivo (Kc), do fator de correção $\left(\mathrm{K}_{\mathrm{L}}\right)$, da intensidade de aplicação (Ia) do sistema, da lâmina bruta (LB) para determinar o tempo de irrigação.

As lâminas de irrigação aplicadas, são de acordo com ETc, enquanto os valores de $\mathrm{Kc}$, utilizados para determinar a ETc, são baseados na fase fenológica de cada cultura.

Para o $\mathrm{K}_{\mathrm{L}}$, devido à aplicação localizada não necessitar irrigar toda a área, foi utilizado o método proposto por Keller \& Bliesner (1990), Equação 1 .

$$
K_{L}=0,1 \sqrt{P}
$$

onde P é a porcentagem da área molhada (PAM).

A ETc localizada então é determinada com a Equação 2:

$$
E T c=E T_{0} * K c * K_{L}
$$

A intensidade de aplicação (Ia) é determinada de acordo com o número de gotejadores $(\mathrm{N})$, vazão de cada gotejador $(\mathrm{q})$ em relação à área ocupada pela planta (A), Equação 3.

$I_{a}=\frac{N \times q}{A}$

Segue-se com a determinação da lâmina bruta (LB) de irrigação, que é uma relação entre ETo e a eficiência de irrigação $\left(E_{\mathrm{i}}\right)$, multiplicado pelo $\mathrm{K}_{\mathrm{L}}$. Após a obtenção da LB, determina-se o tempo de irrigação, conforme Marques (2013) (Equações 4 e 5).

$L B=\frac{E T_{0}}{E_{i}} x K_{L}$

$T i=\frac{L B}{I_{a}}$

\section{Calibração do cálculo de evapotranspiração}

A estimativa da ETo é determinada pelo método de Romanenko, modificado por Oudin et al. (2005), seguindo a Equação 6.

$$
\text { ETo }=4,5\left(1+\left(\frac{\text { Tmed }}{25}\right)\right)^{2} *\left(1-\left(\frac{\mathrm{e}_{\mathrm{a}}}{\mathrm{e}_{\mathrm{s}}}\right)\right) \text { Eq.(6) }
$$

O método foi calibrado e validado pelos autores para a estação agrometeorológica local, para um período de 2 anos de dados de estação agrometeorológica da Onset, ajustando-se à Equação 7.

$\mathrm{ETo}=2,5982\left(1+\left(\frac{\text { Tmed }}{25}\right)\right)^{2} *\left(1-\left(\frac{\mathrm{e}_{\mathrm{a}}}{\mathrm{e}_{\mathrm{s}}}\right)\right)+0,7972$

Eq.(7)

onde $e_{a}$ é a pressão real de vapor $(\mathrm{kPa})$ e $\mathrm{e}_{\mathrm{s}}$ a pressão de saturação de vapor $(\mathrm{kPa})$. Os dois parâmetros são baseados na temperatura média (Tmed) e umidade relativa do ar média (URmed), conforme as Equações 8 e 9:

$\mathrm{e}_{\mathrm{s}}=0,6108 * \exp \left[\frac{17,27 * \mathrm{Tmed}}{\mathrm{Tmed}+237,3}\right]$

$\mathrm{e}_{\mathrm{a}}=\frac{\mathrm{e}_{\mathrm{s}}{ }^{*} \text { URmed }}{100}$

Os resultados de ETo, estimados pelo método PM (Penman-Montheith) FAO 56 (Allen et al., 2005), foram comparados com os de ETo calculada por métodos alternativos, de modo a obter o desempenho localmente. Com base nos valores de ETo, foram ajustadas equações de 
regressão linear simples, onde a variável dependente foi a equação de PM FAO 56, e variável independente os valores de ETo estimados pelos demais métodos.

A equação de Romanenko foi calibrada em relação ao método de PM para a estação agrometeorológica Onset (Modelo - EAC-U30900), localizada em Pesqueira - PE.

A análise estatística do desempenho dos métodos de estimativa de evapotranspiração de referência estudados baseou-se na metodologia descrita por Willmott \& Matsuura (2005) (Equações 10, 11 e 12).

$$
\begin{gathered}
\mathrm{RMSE}=\sqrt{\frac{1}{\mathrm{n}} \sum\left(\mathrm{ETo}_{\text {método }}-\mathrm{ETo}_{\text {PMFAO56 }}\right)^{2}} \\
\text { Eq.(10) } \\
\mathrm{MBE}=\frac{1}{\mathrm{n}} \sum\left(\mathrm{ETo}_{\text {método }}-\mathrm{ETo}_{\text {PMFAO56 }}\right) \\
\text { Eq.(11) }
\end{gathered}
$$

$$
\mathrm{EP}=\frac{\left(\mathrm{ETo}_{\text {método }}-\mathrm{ETopMFAO56}\right)}{\text { ETopMFAO56 }}
$$

onde, $\mathrm{n}=$ número de observações dos valores de ETo $_{\text {FAOPM56 }}$ e $\mathrm{ETo}_{\text {método, que respectivamente, }}$ representam os valores diários de ETo estimados com os dados completos e métodos alternativos; RMSE = raiz quadrada do quadrado do erro médio $\left(\mathrm{mm} \mathrm{dia}{ }^{-1}\right) ; \mathrm{MBE}=$ erro médio de estimativa $(\mathrm{mm}$ dia $\left.^{-1}\right) ; \mathrm{EP}=$ erro percentual de estimativa $(\%) . \mathrm{O}$ RMSE que fornece subsídios para quantificar a sub ou superestimativa dos valores de ETométodo, em relação ao ETopmfaO56, a curto prazo, e o MBE, a longo prazo.

Foram calculados os índices estatísticos de precisão " $r$ " (coeficiente de correlação) e de exatidão "d" (índice de concordância) (Willmott et al., 1985) (Equações 13 e 14).

$$
\begin{aligned}
& \mathrm{r}=\frac{\left[\sum \mathrm{ETo}_{\text {PMFAO56 }} \mathrm{XETo}_{\text {método }}-\left(\frac{\left.\sum \mathrm{ETo}_{\text {PMFAO5 }} \times \sum \mathrm{ETo}_{\text {método }}\right)}{\mathrm{n}}\right)\right.}{\sqrt{\sum\left(\mathrm{ETo}_{\text {PMFAO56 }}-\text { ETo }_{\text {PMFAO56 }}\right)^{2} \times \sum\left(\mathrm{ETo}_{\text {método }}-\text { ETo }_{\text {método }}\right)^{2}}} \\
& \text { Eq.(13) }
\end{aligned}
$$

$$
\mathrm{d}=1-\frac{\sum\left(\mathrm{ETo}_{\text {método }}-\mathrm{ETo}_{\text {PMFAO56 }}\right)^{2}}{\sum\left(\mid \mathrm{ETo}_{\text {método }}-\frac{\left.\mathrm{ETo}_{\text {PMFAO56 }} \mid\right)\left(\left|\mathrm{ETo}_{\text {método }}-\mathrm{ETo}_{\text {PMFAO56 }}\right|\right)^{2}}{\text { Eq. }(14)}\right.}
$$

As barras sobre ETopmfaos6 e ETométodo referem-se ao valor médio dos dados considerados. A precisão é dada pelo coeficiente de correlação " $r$ " que indica o grau de dispersão dos dados entre si. O seu valor varia de $-1 \mathrm{a}+1$, de modo que, quanto mais próximos dos extremos, melhor a correlação da $\mathrm{ETo}_{\text {método }}$ com a EToPadrão. Os sinais indicam se a correlação é negativa ou positiva. A exatidão "d" está relacionada ao afastamento dos valores estimados (ETométodo) em relação aos

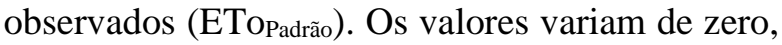
para nenhuma concordância, a 1, para a concordância perfeita.

Para classificar o desempenho do método PM FAO 56 na determinação da ETo com métodos alternativos, foi calculado o índice de confiança (c) conforme Camargo \& Sentelhas (1997), o qual é resultado do produto entre o coeficiente de correlação (r) e o índice de concordância (d) (Tabela 1), conforme a Equação 15.

$c=r x d$

Tabela 1. Critério de interpretação do desempenho dos métodos estimativa da evapotranspiração para a Região do Nordeste brasileiro pelo índice de confiança "c". Fonte: Camargo \& Sentelhas (1997).

\begin{tabular}{cc}
\hline Valor de "c” & Classificação \\
\hline$>0,85$ & Ótimo \\
$0,76-0,85$ & Muito bom \\
$0,66-0,75$ & Bom \\
$0,61-0,65$ & Mediano \\
$0,51-0,60$ & Sofrível \\
$0,41-0,50$ & Mau \\
$\leq 0,40$ & Péssimo \\
\hline
\end{tabular}

Montagem do experimento para avaliação da qualidade das informações meteorológicas

A avaliação da eficiência do sensor de temperatura e umidade relativa do ar foi realizada no Agreste de Pernambuco (Figura 4), em Região de clima classificado como As (com o verão seco) de acordo com a classificação de Köppen (Alvares et al., 2013). 




Figura 4. Localização da montagem do experimento. Fonte: Carvalho et al. (2020).

O sensor de temperatura e umidade do ar DHT-22 foi comparado ao Sensor de Temperatura e Umidade do Ar PLUG-AND-PLAY S-THBM002 (mais descrições em: https://sigmasensors.com.br/produtos/sensor-detemperatura-e-umidade-do-ar-plug-and-play-sthb-m002).

O sensor DHT-22 foi instalado no abrigo meteorológico da microestação, conforme descrito na seção anterior. O sensor S-THB-M002, foi acoplado a uma estação agrometeorológica Onset (Modelo - EAC-U30-900) e acondicionado em um abrigo meteorológico. Ambos os sensores foram

\section{Resultados e Discussão}

O comportamento da evapotranspiração de referência, estimado pela equação de Romanenko, sem a devida calibração, apresentou elevada superestimava, como observado pelos índices EP $(50,8 \%), \operatorname{MBE}\left(2,78 \mathrm{~mm} \mathrm{dia}^{-1}\right), \operatorname{RMSE}(3,17$

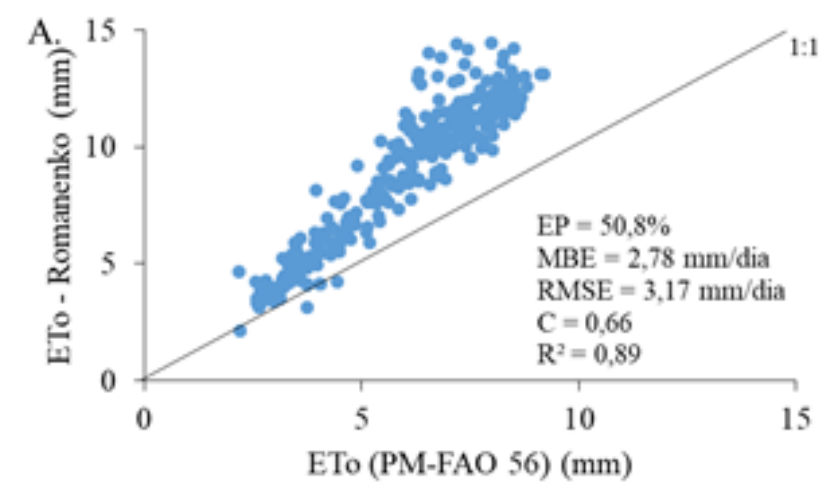

instalados à altura padrão de 2 metros do solo, próximos um do outro, preservando as mesmas condições para ambos.

Os dados das médias e desvio padrão para os horários de temperatura do ar e umidade do ar que foram capturados por cada um dos sensores foram comparados através de análise gráfica. Além disso, uma regressão linear entre às duas variáveis (assumindo a coleta do DHT22 como variável independente e a coleta do S-THB-M002 como variável dependente) foi realizada de modo a observar o coeficiente de determinação $\left(\mathrm{R}^{2}\right)$ e o grau de similaridade entre os resultados obtidos. $\mathrm{mm} \operatorname{dia}^{-1}$ ) e pelo índice de confiança $\mathrm{c}=0,66$, classificado como bom. Em relação ao uso do método calibrado, constatou-se boas estimativas, com baixo EP (1,64\%), MBE $\left(0,04 \mathrm{~mm} \mathrm{dia}^{-1}\right)$, RMSE (0,64 mm dia $\left.{ }^{-1}\right)$ e um ótimo desempenho (c $=0,91)$, de acordo com o índice de confiança (Figura 5).

Figura 5. Comparação da ETo obtida pelos métodos de Penman-Monteith e de Romanenko sem calibração (A) e com calibração (B). Fonte: Carvalho et al. (2020).

Observou-se uma boa correlação entre os métodos de estimativa da ETo, como coeficiente de determinação de 0,89 para os seis meses de dados

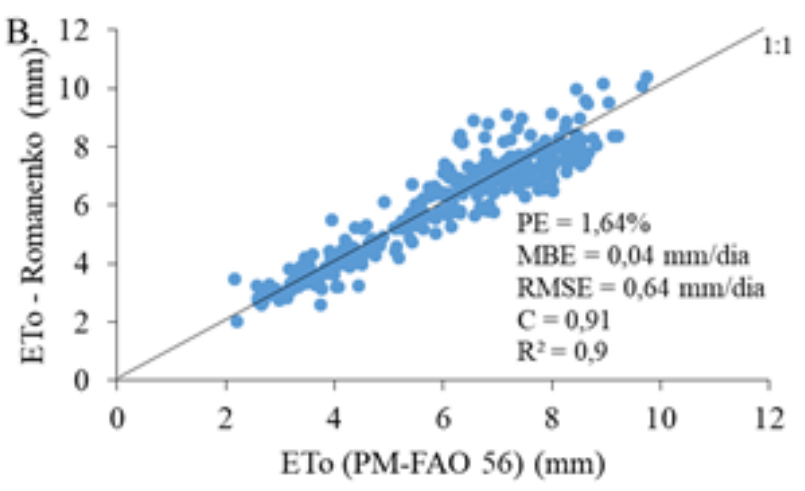

de correlação (Figura 5A) e 0,9 para os seis meses de dados para a calibração (Figura 5B).

Em estudo realizado no Senegal, Djaman et al. (2015) constataram um ótimo desempenho da 
ETo, comparando o método de PM-FAO 56, com o de Romanenko, obtendo-se um $\mathrm{R}^{2}$ de 0,92.

A temperatura coletada pelo sensor da estação simplificada (Temp-Simpl) apresentou comportamento horário muito semelhante ao da estação padrão (Temp-Padrão) (Figura 6A). Em relação à umidade relativa do ar (UR), apesar de ter apresentado o mesmo comportamento quanto ao horário, os valores foram mais discrepantes, com uma superestimativa da UR, principalmente durante os horários mais quentes do dia (Figura 6B). Desta forma, é recomendado a calibração para aproximação dos valores horários.

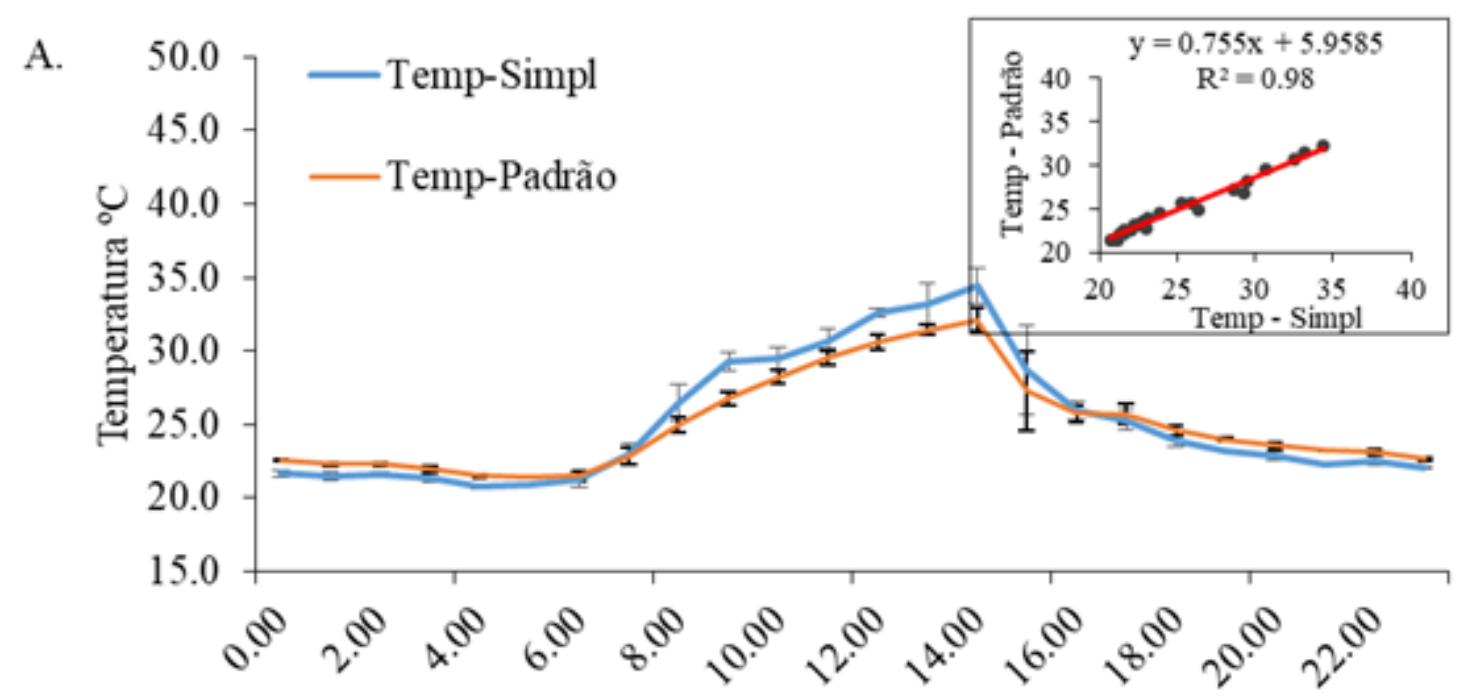

Hora

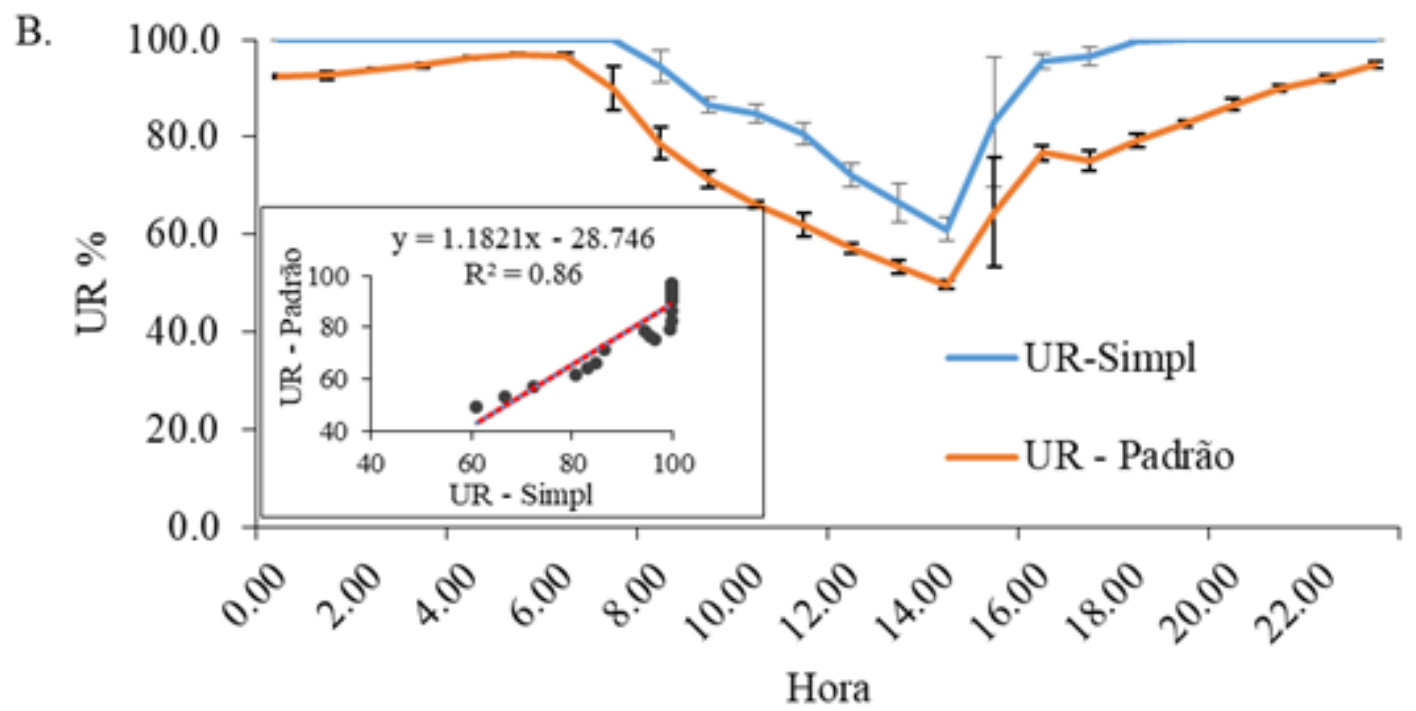

Figura 6. Comportamento horário e correlação da temperatura (Temp) (A) e da umidade relativa do ar (UR) (B) para a estação simplificada (Simpl) e a Padrão. Fonte: Carvalho et al. (2020).

Ótimo coeficiente de determinação foi observado para a temperatura e umidade relativa do ar, com $\mathrm{R}^{2}$ de 0,98 e 0,86 , respectivamente. Comparando-se um sensor de baixo custo (DHT22) no estado de Mato Grosso, Silva Filho et al. (2019) encontraram altos coeficientes de determinação $\left(R^{2}>0,9\right)$ entre a temperatura e a umidade relativa do ar.
A Tabela 2, apresenta os dados da temperatura média (T-med), máxima (T-max), mínima (T-min), a umidade relativa média (URmed), máxima (UR-max), mínima (UR-min) e os desvios da temperatura (T-Desvio) e a umidade do ar (UR-Desvio). Além disso, é apresentada a ETo diária para o método de Romanenko, sem a calibração (ETo-R) e com a calibração (ETo-RCalib). 
Tabela 2. Parâmetros estatísticos da temperatura (T) e umidade relativa do ar (UR) para a estação simplificada (Simpl) e a padrão e valores médios de evapotranspiração de referência obtidos pelo método de Romanenko sem (ETo-R) e com calibração (ETo-R-Calib). Fonte: Carvalho et al. (2020).

\begin{tabular}{ccccccccccc}
\hline $\begin{array}{c}\text { Estaçã } \\
\text { o }\end{array}$ & $\begin{array}{c}\text { T- } \\
\text { med }\end{array}$ & $\begin{array}{c}\text { T- } \\
\text { max }\end{array}$ & $\begin{array}{c}\text { T- } \\
\text { min }\end{array}$ & $\begin{array}{c}\text { T- } \\
\text { Desvio }\end{array}$ & $\begin{array}{c}\text { UR- } \\
\text { med }\end{array}$ & $\begin{array}{c}\text { UR- } \\
\text { max }\end{array}$ & $\begin{array}{c}\text { UR- } \\
\text { min }\end{array}$ & $\begin{array}{c}\text { UR- } \\
\text { Desvio }\end{array}$ & $\begin{array}{c}\text { ETo- } \\
\text { R }\end{array}$ & $\begin{array}{c}\text { ETo-R- } \\
\text { Calib }\end{array}$ \\
\hline Simpl & 25,2 & 35,4 & 20,5 & 4,3 & 92,5 & 99,9 & 59,2 & 11,9 & 3,7 & 2,9 \\
\hline Padrão & 25,0 & 33,0 & 21,1 & 3,3 & 80,6 & 97,3 & 48,4 & 15,0 & 3.5 & 2,8 \\
\hline
\end{tabular}

As diferenças de temperatura T-med e Tmax obtidas pelos sensores Padrão e Simpl foram de $0,2{ }^{\circ} \mathrm{C}$ e $2,4{ }^{\circ} \mathrm{C}$, respectivamente, e os valores menores foram obtidos pelo sensor Simpl. A T-min foi superior a $0,6^{\circ} \mathrm{C}$ na estação Padrão. Observouse um menor desvio padrão $(3,3)$ na temperatura média, na estação Padrão. Em relação à UR, observou-se maiores valores na estação Simpl, com UR-med, UR-max e UR-min superior a, 11,9; 2,6 e $10,8 \%$, respectivamente. Um maior desvio $(15,0)$ foi contatado na UR da estação padrão.

Os maiores valores de evapotranspiração de referência foram observados com os dados climáticos da estação simplificada (Simpl). A diferença da ETo-R da Simpl, comparada ao padrão foi de $0,2 \mathrm{~mm}$, para o uso da fórmula de Romanenko não calibrada e, quando calibrada, a diferença foi de $0,1 \mathrm{~mm}$.

Silva Filho et al. (2019) recomendam o uso do sensor simplificado para o estudo microclimático, porém são necessários estudos futuros que avaliem a durabilidade e a qualidade das informações agroclimáticas.

\section{Conclusão}

ótimo desempenho na estimativa da evapotranspiração de referência pelo método de Romanenko calibrado.

O sensor de temperatura e umidade relativa do ar apresentou alta eficiência nas leituras horárias, principalmente para a temperatura.

Sendo assim, é possível afirmar que a microestação climática desenvolvida neste trabalho pode ser utilizada com alto grau de confiança no processo de automação de irrigação. Como trabalho futuro planeja-se mensurar o grau de degradação dos sensores ao longo do tempo.

\section{Agradecimentos}

Os autores agradecem ao Conselho Nacional de Desenvolvimento Científico e Tecnológico-CNPq, à Coordenação de Aperfeiçoamento de Pessoal de Nível SuperiorCAPES, ao projeto Aliança para Gestão de Recursos Hídricos no Semiárido de Pernambuco (Consórcio UNIVERSITAS) financiado pela Fundação de Amparo a Ciência e Tecnologia do
Estado de Pernambuco-FACEPE (Processo $\mathrm{N}^{\circ}$. APQ-0300-5.03/17).

\section{Referências}

Agrawal, A.; Kamboj, V.; Gupta, R.; Pandey, M.; Tayal, V. K.; Singh, H. P. 2018. Microcontroller Based Irrigation System Solar Powered Using Moisture Sensing Technology. In: 2018 8th International Conference on Cloud Computing, Data Science \& Engineering (Confluence). IEEE, 324-327.

DOI: 10.1109/CONFLUENCE.2018.8442551

Agrawal, N.; Singhal, S. 2015. Smart drip irrigation system using raspberry pi and arduino. In: International Conference on Computing, Communication \& Automation. IEEE, 928-932. DOI: 10.1109/CCAA.2015.7148526

Allen, R. G.; Walter, I. A.; Elliott, R.; Howell, T. A.; Itenfisu, D.; Jensen, M. E.; 2005. The ASCE standardized reference evapotranspiration equation [WWW Document]. EPIC3Idaho Task Comm. Stand. Ref. Evapotranspiration.

Alvares, C. A.; Stape, J. L.; Sentelhas, P. C.; Moraes, G.; Leonardo, J.; Sparovek, G. 2013. Köppen's climate classification map for Brazil. Meteorologische Zeitschrift, 22, 711728. DOI: 10.1127/0941-2948/2013/0507

Camargo, A. P.; Sentelhas, P. C.; 1997. Avaliação do desempenho de diferentes métodos de estimativa da evapotranspiração potencial no Estado de São Paulo, Brasil. Revista Brasileira de Agrometeorologia, 5, 89-97.

Djaman, K.; Balde, A. B.; Sow, A.; Muller, B.; Irmak, S.; N'Diaye, M. K.; Mannel, B.; Moukoumbi, Y. D.; Futakuchi, K.; Saito, K. 2015. Evaluation of sixteen reference evapotranspiration methods under sahelian conditions in the Senegal River Valley. Journal of Hydrology: regional studies, 3, 139-159. DOI: 10.1016/j.ejrh.2015.02.002

Duarte, A. Reuso de água residuária tratada na irrigação da cultura do pimentão (Capsicumannun L). 2006. Escola Superior de Agricultura Luiz de Queiroz, Piracicaba, $187 \mathrm{p}$.

Keller, J.; Bliesner, R. D. 1990. Sprinkle and trickle 
irrigation. New York: Avibook, 649p.

Lopes, A. S.; Pavani, L. C.; Corá, J. E.; Zanini, J. R.; Miranda, H. A. 2004. Manejo da irrigação (tensiometria e balanço hídrico climatológico) para a cultura do feijoeiro em sistemas de cultivo direto e convencional. Engenharia Agrícola, Jaboticabal, 24, 89$100 . \quad$ DOI: $\quad 10.1590 / \mathrm{S} 0100-$ 69162004000100011

Melo, T. K.; Medeiros, J. F.; Sobrinho, J. E.; Figueiredo, V. B.; Souza, P.S. 2013. Evapotranspiration and crop coeficientes of melon plants measured by lysimeter and estimated according to fao 56 methodology Engenharia Agrícola, Jaboticabal, 34, 929$939 . \quad$ DOI: $\quad 10.1590 / \mathrm{S} 0100-$ 69162013000500005

Oudin, L.; Hervieu, F.; Michel, C.; Perrin, C.; Andréassian, V.; Anctil, F.; Loumagne, C. 2005. Which potential evapotranspiration input for a lumped rainfall-runoff model?: Part 2 - Towards a simple and efficient potential evapotranspiration model for rainfall-runoff modelling. Journal of Hydrology, 303, 290-306. DOI: 10.1016/j.jhydrol.2004.08.025

Pereira Filho, I. A.; Alvarenga, R. C.; Gontijo Neto, M. M.; Viana, J. H. M.; Oliveira, M. F. 2010. Cultivo do milho. 6 Ed. Sete Lagoas: Embrapa Milho e Sorgo. 10p.

Silva Filho, A.; Biudes, M. S.; Machado, N. G.;
Querino, C. A. S.; Júnior, E. S. A. 2019. Estimativa do balanço de energia pelo método da razão de Bowen a partir de medidas de protótipo de estação micrometeorológica. Revista Brasileira de Climatologia, 24, 134-150.

Uddin, J.; Reza, S. T.; Newaz, Q.; Uddin, J.; Islam, T.; Kim, J. M. 2012. Automated irrigation system using solar power. In: 2012 7th International Conference on Electrical and Computer Engineering. IEEE, 228-231. DOI: 10.1109/ICECE.2012.6471527

Willmott, C. J.; Ackleson, S. G.; Davis, R. E.; Feddema, J. J.; Klink, K. M.; Legates, D. R.; O'Donnell, J.; Rowe, C. M. 1985. Statistics for the evaluation and comparison of models. J. Geophys. Res. Oceans 90, 8995-9005. DOI: 10.1029/JC090iC05p08995

Willmott, C. J.; Matsuura, K.; 2005. Advantages of the mean absolute error (MAE) over the root mean square error (RMSE) in assessing average model performance. Climate Research, 30, 79-82. DOI: 10.3354/cr030079

Trilles, S.; Torres-Sospedra, J.; Belmonte, Ó.; Zarazaga-Soria, F. J.; González-Pérez, A.; Huerta, J. 2019. Development of an open sensorized platform in a smart agriculture context: A vineyard support system for monitoring mildew disease. Sustainable Computing: Informatics and Systems. 30p. DOI: 10.1016/j.suscom.2019.01.011 